\title{
The Big 5 at the University of Kansas Medical Center: Remaining Competitive in Today's Research Environment
}

\author{
Paul F. Terranova
}

Vice Chancellor for Research, University of Kansas Medical Center

$\mathrm{T}$

The term 'Big 5' was established as the five established research areas at the University of Kansas Medical Center and includes Cancer, Reproductive Sciences, Neurosciences, Kidney and Liver. Each of these areas is part of the $10-y r$ vision to enhance and sustain life sciences research at KUMC as described in 'The Time is Now' (http://www.kumc.edu/evc/TheTimeIsNow.pdf) and each is an established disease or organ-based Center or an Institute at the Medical Center. However, Cancer, Neurosciences and Liver include faculty at the University of Kansas at Lawrence and other institutions throughout the region.

Each of these Centers and Institutes has common characteristics including 1) a Founder and/or Director with significant accomplishments nationally and internationally and a desire to grow the program 2) Shared Resources that support the research programs 3) Program Grants 4) Numerous pre- and postdoctoral students 5) Consolidated research space 6) External Advisory Board 7) Collaborations 8) Seminar Program and 9) Outreach. Each of these nine areas will be discussed and then a summary of each Center and Institute will be provided.

\section{Founder and/or Director}

Each of the Big 5 has a founder and/or a director with significant accomplishments nationally and internationally and a desire to conceive and build new programs and grow existing programs, facilitate research by promoting collaborations and development and use of shared resources, recruit, train and mentor outstanding students and faculty, and promote basic as well as clinical and translational research. Although each of these founder/director characteristics is important, each is not necessarily equal in importance. In fact, the relative importance of each founder/director characteristic is center/institute-dependent. For example, at the time of establishment of a center or institute, leadership qualities including a nationally and internationally recognized scientist may be quite important in order to impart a high degree of visibility that would help in recruiting outstanding faculty and students. During the established years, when the number of faculty is at equilibrium, facilitating research collaborations and developing new shared resources may quite important.

\section{Shared Resources}

Each of the Big 5 has shared resources that support research programs within each Center/Institute as well as noncenter/institute members throughout the 
university. An important approach in developing and sustaining shared resources is to continually monitor the research needs of center/institute members. Shared resources such as DNA sequencing may been heavily used 20 years ago but outsourcing of this technology has become more cost effective. Examples of some cores within our university include grant preparation and evaluation, laboratory reagent procurement, medical illustration and drawing, microscopy (electron, laser capture, fluorescent), genomics, proteomics, gene targeting and mouse transgenics, animal behavior assessment and flow cytometry.

\section{Program Grants}

Each of the Big 5 has program grants, especially from NIH including collaborative research projects such as U54, P01 and P50 and core based grants (P30). The collaborative research projects usually include the majority of project leaders from within the university but subcontracts are also signed with other collaborative universities. The collaborative research projects (U54, P01 and P50) have research projects similar to R01 grants but each project is related and collaborative. Centers/institutes support by collaborative research grants have a significant number of established investigators in the field. NIH P30 grants support shared resources and are designed to meet the needs of investigators within a specific program, center or institute. P30 grants include a significant portfolio of R01 grants that require the usage of the shared resources. NIH training grants (T32) include stipend and travel support for pre- and/or postdoctoral fellows. T32 training grants are awarded to those universities that have a significant number of NIH R01 funded investigators that can serve as mentors to the trainees. T32 training grants are indicative of a mature program. Some of the Big 5 programs have NIH K12 grants that support the development of junior faculty researchers. The K12 provides significant salary support for the junior faculty investigators as well as a research supply budget. Each of the Big 5 also has multiple R01 grants and private foundation grants.

\section{Numerous pre- and postdoctoral students \\ Each of the Big 5 is continually} recruiting students through networking and advertisements at national and international meetings. Although some students may be admitted to the graduate or postdoctoral program on institutional training grants, when possible, students are required to apply for individual pre- or post-doctoral fellowships. All of the students are integrated into the activities of the center/institute including participating on committees, interviewing prospective students and faculty, giving seminars, and mentoring their junior colleagues. Students are expected to participate in the local and national activities of the center/institute by attending scientific meetings and participating in student led organizations associated with various societies.

\section{Consolidated space}

Each of the Big 5 have consolidated space including laboratories, shared resources, offices and administrative area. For example, The Cancer Center has 140,000 sq. $\mathrm{ft}$ of consolidated space whereas the Reproductive and Kidney Centers have 20,000 sq. ft. each and the Institute of Neurological Disorders and the Liver Center have 14,000 sq. $\mathrm{ft}$. each. Within the center/institute consolidated space promotes scientific interactions and the resulting 
collaborations can be significant in the form of joint grants and publications and sharing resources. In addition, the provision of services to the center/institute membership in a central location results in a high degree of efficiency by reducing duplication of resources. Consolidated research and administration also increases the visibility of the center/institute within the university and for invited guests, e.g., seminar speakers, external advisors and review teams.

\section{External Advisory Board}

Each of the Big 5 have nationally prominent scientists that assess the overall organization of the center/institute, and its vision and goals, progress, scientific direction and impact, centeredness, and the efficiency of the cores. External advisory board members are also very helpful in reviewing program grants prior to submission to the $\mathrm{NIH}$ or other granting agency. Collectively, the external advisors have specific scientific or technical expertise that is components of the center/institute. External advisors also provide assistance in recruiting faculty and students.

\section{Collaborations}

Each of the Big 5 has well-established collaborations within the center/institute and university as well as with other universities. Thus, collaborations may be local, national, and international and involve students and faculty that has joint publications and grants and share technologies

Seminar Program including an Annual Symposium

Each of the Big 5 has a seminar program and an annual symposium /workshop. The center/institute invites local, national and international experts who are the plenary speakers. Local students are also invited to speak at the podium, lead sessions and present posters. A student or faculty member within the center/institute is generally assigned to a plenary speaker to assure their needs are met including transportation, meals, and introductions to other guests. This concierge type service works extremely well for our guests and assures a successful visit. The seminar program and annual symposium increases the visibility of the center/institute, an invaluable component.

\section{Outreach}

Each of the Big 5 is involved in outreach that includes other centers /institutes and departments within the university, and the local, national, and international communities. Outreach has an educational component that provides information about the activities of the center/institute as well as a fund raising component to support specific initiatives. Some of the centers/institutes have a newsletter that keeps interested parties informed of their activities.

The following is a brief summary of the goals of each of the Big 5 .

\section{University of Kansas Cancer Center} (http://cancer.kumc.edu/)

The University of Kansas Cancer Center under the Directorship of Roy Jensen, MD has three goals:

Establish a critical mass of basic, translational, and clinical cancer researchers whose scientific productivity will drive the drug discovery, delivery, and development process.

Develop and sustain a Cancer Center culture, infrastructure, and state-of-the-art research facilities that foster the translation of scientific findings into new cancer therapies, and enhanced prevention, control, and survivorship strategies. 
Ensure that our cancer drug innovation pipeline benefits cancer patients throughout our region and becomes the model for cancer drug discovery in academia.

The programs with the Cancer Center are Drug Discovery, Development and Delivery, Cancer Biology, Cancer Prevention, and Cancer Control and Public Health.

Institute for Neurological Disorders (http://www.indkc.org/)

The goal of the Institute for Neurological Disorders is to advance neuroscience translational research (bench to bedside) and thus, taking what is discovered in basic science labs and applying it to improve clinical outcomes. The mechanisms by which this is accomplished is by 1) communication, initiating and promoting dialogs between clinicians and basic researchers, 2) coordination, promoting interaction between clinical programs and basic science research, and 3) consolidation, creating teams of basic and clinical researchers with common interests and goals. The Institute has several divisions including Brain Injury and Repair, Neuromuscular and Movement Disorders, Neurodegenerative Disorders, Hearing and Equilibrium Disorders, Female Pain Syndromes, and Cognitive and Behavioral Neuroscience.

Institute for Reproductive Health and Regenerative Medicine (website under development)
The goal of the Institute is to facilitate investigator and especially multiinvestigator research initiatives in basic, translational, and clinical research directed toward reproductive health and regenerative medicine. There are four centers within the Institute including Reproductive Health, Maternal-Fetal Biology, Contraceptive \& Drug Development, and Stem Cells \& Epigenetics.

\section{Kidney Institute}

\section{(http://www2.kumc.edu/ki/)}

The mission of the Kidney Institute is to promote research leading to a better understanding of renal disease and the development of clinical trials ultimately for improved patient outcomes. Research areas include Polycystic Kidney Disease, Glomerular \& Vascular Disease, Kidney Development, Chronic Kidney Disease, Bone-Kidney Interactions, and Kidney Cancer.

\section{Liver Center}

(http://www.kumc.edu/livercenter/donate. html)

The mission of the Liver Center is to bring together, on a cooperative basis, basic science and clinical investigators to enhance the effectiveness of conducting translational liver research. Research programs include nuclear receptor biology, hepatic metabolism/ biotransformation, liver injury and cell growth regulation (cancer, regeneration). 